\title{
Threshold selection method for UWB TOA estimation based on wavelet decomposition and kurtosis analysis
}

\author{
Juan $\mathrm{Li}^{1}$, Xuerong Cui ${ }^{1 *}$ D, Houbing Song ${ }^{2}$, Zhongwei Li ${ }^{1}$ and Jianhang Liu ${ }^{1}$
}

\begin{abstract}
In wireless sensor networks, ranging or positioning via ultra-wideband (UWB) has caused widespread research interests where the non-coherent energy detection (ED) method with low sampling rate and low complexity is widely studied. However, the traditional energy detection methods only analyze the signal energy in the time domain, so their error is relatively large. In this paper, the simulation results show that most of the signal energy concentrates in the low-frequency band, so a novel threshold selection method for time of arrival (TOA) estimation is proposed that analyzes the signals in both time domain and frequency domain. In this method, the received signal is decomposed by "db6" wavelet and the kurtosis of energy blocks of the low-frequency wavelet coefficients $\left(K_{c}\right)$ is analyzed. At last, the mapping relationship between $K_{c}$ and the normalized threshold for TOA estimation is created using polynomial fitting with degree 3 . The simulation results show that the TOA estimation error of the proposed method is significantly less than the method without wavelet decomposition.
\end{abstract}

Keywords: Ranging, Wavelet decomposition, Kurtosis analysis, Ultra-wideband, Energy detection, Threshold selection

\section{Introduction}

In recent years, with the development of wireless communication technologies $[1,2]$, the applications of wireless sensor networks are more and more widely used. The important premise of these applications is to obtain the precise position of the targets [3-5]. Therefore, the precise positioning of targets becomes the key problem to be solved urgently.

Ultra-wideband (UWB) is a new wireless communication technology [6-9], which is widely used in many fields, such as indoor short-distance communication, high-speed wireless local area networks (WLAN) [10], security monitoring, ranging, positioning, and so on. UWB is the most promising technology for indoor positioning and tracking [3]. Compared with other shortrange communication technology, UWB has many advantages for short-range communication: first, UWB can provide up to $\mathrm{GHz}$ bandwidth; second, UWB can provide data rates of hundreds of megabits per second

\footnotetext{
* Correspondence: cxr@upc.edu.cn

${ }^{1}$ Department of Computer and Communication Engineering, China

University of Petroleum (East China), Qingdao 266580, China

Full list of author information is available at the end of the article
}

or even gigabits per second, so it is an ideal technology for wireless communication in wireless sensor networks [11, 12]; third, continuous transmission carrier is not needed in UWB communication, and the intermittent pulse is used to transmit data, which make short pulse duration, low power consumption, and high multipath resolution.

Wireless positioning methods can be divided into fingerprint positioning algorithm based on received signal strength indicator (RSSI) [13], geometric or range positioning algorithm based on the range, time of arrival (TOA) [14, 15], time difference of arrival (TDOA) [16], or angle of arrival (AOA) [17], and some fusion positioning methods together with inertial measurement units (IMUs) [5]. The signal fingerprint positioning algorithm $[4,18]$ is based on the mapping relationship between some parameters obtained from the received signal and the position information of the target node. The range-based positioning algorithm with round-triptime (RTT) measurements [19] is often used to meet the requirement of high-precision positioning because of its high time delay resolution. However, obtaining the accurate ranging estimation is a very challenging 
problem due to the effects of thermal noise, multi-path fading, non-line of sight, and other factors in wireless transmission channel. For example, in non-line of sight (NLOS) environment, the range estimation based on TOA will typically be positively biased [3].

In recent years, ranging algorithms for UWB systems have been extensively studied. There are three main approaches. The first approach is matched filter (MF) based on coherent algorithm with high sampling rate [20]. The second is machine learning method based on some selected channel parameters. In [3], a ranging method based on kernel principal component is proposed, where the channel parameters are projected onto a high-dimensional nonlinear orthogonal space, and then the subset from these projections is used for ranging. The third is energy detection (ED) algorithm based on non-coherent receiver with low sampling rate and low complexity $[8,9,21]$. The matched filter approach is not applicable in many practical situations due to the high complexity and high hardware requirement. As opposed to the complex matched filter method, the energy detection is a non-coherent method for TOA estimation which consists of a square-law device, an integrator, a sampler, and a decision mechanism. The TOA value is estimated by the first signal sample exceeding a specific threshold which is deemed as the start of the received signal. Thus, the energy detection method is applicable in many cases because it is a method with low complexity and low sampling rate. In this method, how to select an appropriate threshold is a key issue. In literature [9], a threshold selection method based on kurtosis analysis of energy blocks was proposed, and in literature [21], a threshold selection method based on skewness analysis of energy blocks was also put forward. However, the TOA estimation accuracy of these methods is not very high because these parameters such as kurtosis of the received signals can only reflect statistical characteristics in time domain and ignore all the characteristics in frequency domain. At the same time, the received signals will be affected by the random noise, so the large randomness will result in the poor precision of kurtosis in time domain.

In this paper, the simulations of UWB signal spectrum under different signal to noise ratio (SNR) find out that the UWB signal energy is mainly distributed in the lowfrequency band, while the energy of the white Gauss noise is evenly distributed over the entire frequency band. The wavelet transform is equivalent to two channel filter banks with low-pass and high-pass characteristics, so using the wavelet transform, most of the signal energy concentrates in the low-frequency coefficients, while the energy of white Gauss noise distributes in the coefficients of all frequency bands. Thus, in this paper, after the wavelet transform used in the received signal, the high-frequency coefficients are discarded, and only the low-frequency coefficients are used as the received signal energy to improve the accuracy of ranging. In this way, the white Gauss noise interference in the signal can be reduced effectively.

The remainder of this paper is organized as follows. In Section 2, the UWB ranging system model is presented. Section 3 discusses some ranging estimation algorithms based on traditional energy detection method in the time domain. Section 4 introduces the proposed threshold selection method for TOA estimation based on wavelet decomposition, energy detection, and kurtosis analysis. In Section 5, the simulation results and the performance discussion are presented, and Section 6 concludes the paper.

\section{UWB ranging system models}

\subsection{Pulse waveform}

In UWB ranging system, short pulses with sharp rising and falling edges are usually used as the transmitting signal to get shorter pulse duration (nanosecond level) or higher time delay resolution.

The second derivative of Gauss function is in accord with this characteristic, so it is used as the UWB pulse signal, which can be expressed as Eq. (1)

$$
f(t)=\frac{d^{2} q(t)}{d t^{2}}=\left(1-4 \pi \frac{t^{2}}{\alpha^{2}}\right) e^{-\frac{2 \pi t^{2}}{\alpha^{2}}}
$$

where $q(t)$ is the Gaussian pulse, $\alpha$ denotes the shape factor of the waveform, and a smaller value of $\alpha$ results in a shorter pulse.

\subsection{Modulation method}

In order to improve the capacity of anti-interference, pulse position modulation (PPM) and time hopping spread spectrum (TH-SS) [7] are used which can be expressed as Eq. (2)

$$
s(t)=\sum_{i} f\left(t-i T_{f}-c_{i} T_{c}-a_{i} \varepsilon\right)
$$

where frame index and frame duration are denoted by $i$ and $T_{f}$ respectively, $T_{c}$ is the chip duration, hopping sequence is composed of integer-valued $c_{i} \in\left\{0,1, \ldots, T_{f} / T_{c}\right.$ $-1\}, a_{i}$ is a binary sequence, and $\varepsilon$ is the time shift in pulse position modulation.

\subsection{IEEE 802.15.4a channel model}

The simulations in this paper are done in the IEEE802.15.4a channel model [22]. IEEE802.15.4a [22] is the first international standard for ranging and positioning in physical layer which provides high-accuracy ranging and positioning capability. The IEEE 802.15.4a standard specifies two signaling formats based on 
impulse radio ultra-wideband (IR-UWB) and chirp spread spectrum (CSS). The former is used for ranging, and the latter is used for wireless communication. Since this paper is focused on TOA estimation or ranging algorithm, therefore, only the IR-UWB is considered in line of sight (LOS) and NLOS environments. Classification of channel models in IEEE 802.15.4a are listed in in Table 1.

CM1 and CM2 are the indoor residential channel models with LOS and NLOS environments which are used for the simulations in this paper. The channel impulse response can be expressed as Eq. (3)

$$
h(t)=\sum_{l=1}^{L} \sum_{k=1}^{K(l)} \alpha_{l, k} \delta\left(t-T_{l}-\tau_{l, k}\right)
$$

where $L$ is the number of received clusters, $K(l)$ denotes the number of received multipath components in the $l$ th cluster, $\alpha_{l, k}$ is the gain factor of the $k$ th multipath component of the $l$ th cluster, $T_{l}$ denotes the TOA of the $l$ th cluster, and $\tau_{l, k}$ is the TOA of the $k$ th multipath component of the $l$ th cluster. The environments of CM1 and CM2 are shown in Table 2.

The received signal can be expressed as Eq. (4)

$$
r(t)=s(t) \times h(t)+n(t)
$$

where $n(t)$ is the additive white Gaussian noise (AWGN) with zero mean and two-sided power spectral density $N_{0} / 2$. Thus, the received signal can be expressed as Eq. (5)

Table 1 Classification of channel models specified in IEEE 802.15.4a standard

\begin{tabular}{ll}
$\begin{array}{l}\text { Channel } \\
\text { models }\end{array}$ & Channel description \\
\hline CM1 & $\begin{array}{l}\text { Residential environment with LOS communication } \\
\text { (7-20 m) }\end{array}$ \\
CM2 & $\begin{array}{l}\text { Residential environment with NLOS communication } \\
\text { (7-20 m) }\end{array}$ \\
CM3 & $\begin{array}{l}\text { Office environment with LOS communication } \\
\text { (3-28 m) }\end{array}$ \\
CM4 & $\begin{array}{l}\text { Office environment with NLOS communication } \\
\text { (3-28 m) } \\
\text { CM5 }\end{array}$ \\
Outdoor environment with LOS communication \\
(5-17 m) \\
Outdoor environment with NLOS communication \\
(5-17 m) \\
CM7
\end{tabular}

Table 2 Channel parameters of CM1 and CM2

\begin{tabular}{lll}
\hline Channel parameters & $\begin{array}{l}\text { CM1 } \\
\text { (LOS) }\end{array}$ & $\begin{array}{l}\text { CM2 } \\
\text { (NLOS) }\end{array}$ \\
\hline $\begin{array}{l}\text { Frequency dependency of the channel } \\
\begin{array}{l}\text { Standard deviation of the log-normal } \\
\text { shadowing of entire impulse response }\end{array}\end{array}$ & 1.12 & 1.53 \\
Mean number of clusters & 3 & 3.51 \\
Cluster arrival rate & 0.047 & 3.5 \\
$\begin{array}{l}\text { Two ray arrival rates (rays per nanosecond) } \\
\text { for mixture of Poisson processes }\end{array}$ & $1.54,0.15$ & $1.77,0.15$ \\
Cluster decay factor (time constant, ns) & 22.61 & 26.27 \\
\hline
\end{tabular}

$$
r(t)=\sum_{l=1}^{L} \sum_{k=1}^{K(l)} \alpha_{l, k} s\left(t-T_{l}-\tau_{l, k}\right)+n(t)
$$

\subsection{TOA estimation error}

The mean absolute error (MAE) of TOA estimation results is expressed as Eq. (6)

$$
\text { MAE }=\frac{1}{N} \sum_{n=1}^{N}\left|t_{n}-\hat{t}_{n}\right|
$$

where $t_{n}$ is the real TOA, $t_{n}$ denotes the estimated TOA, and $N$ is the simulation times.

\section{Energy detection method}

As shown in Fig. 1, in the traditional energy detection methods, the received signal is squared by the low noise amplifier, and then input into an integrator with period $T_{b}$ which is much longer than the sampling interval, so the number of energy blocks within a frame is $N_{b}=\left\lfloor T_{f}\right\rfloor$ $\left.T_{b}\right\lrcorner$ ( \llcorner\lrcorner denotes the integer part) and $T_{f}$ is the frame period.

Thus, the sample values of integrator output are given by Eq. (7)

$$
z[n]=\sum_{j=1}^{N_{p}} \int_{(i-1) T_{f}+\left(c_{j}+n-1\right) T_{b}}^{(i-1) T_{f}+\left(c_{j}+n\right) T_{b}}|r(t)|^{2} d t
$$

where $n=1,2, \ldots, N_{\mathrm{b}-1}, N_{\mathrm{b}}$ is the received sample index in each integration period, and $N_{p}$ is the pulse number in one symbol. In this paper, $N_{p}=1$, so the sample values of integrator output are given by Eq. (8)

$$
z[n]=\int_{(i-1) T_{f}+(n-1) T_{b}}^{(i-1) T_{f}+n T_{b}}|r(t)|^{2} d t
$$

There are many TOA estimation methods based on the energy blocks $z[n]$ which can detect the start of a received signal or estimate the TOA. The simplest one is the maximum energy method, which chooses the maximum energy block $z[n]$ as the first received signal and 


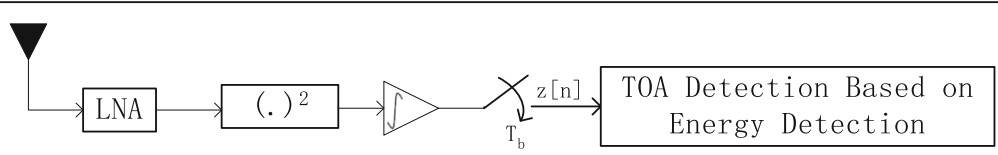

Fig. 1 Block diagram of the energy detection method

the TOA is equal to time responding to the center of the block. However, as shown in Fig. 2, usually, the maximum $z[n]$ is not responding to the first received signal; in this case, the estimated TOA will have big error, especially in NLOS environments. Thus, threshold crossing method has been proposed; with this method, the received energy blocks $z[n]$ are compared with a selected threshold. In this case, the TOA estimation can be obtained according to the first threshold exceeding sample index, which is expressed as Eq. (9)

$$
\hat{t}_{T C}=[\min \{n \mid z[n]>\xi\}-0.5] T_{\mathrm{b}},
$$

It is very difficult to determine an appropriate threshold $\xi$ directly because if the big change of energy and signal, so a normalized threshold $\xi_{\text {norm }}$ ranging from 0 to 1 is often used which is expressed as Eq. (10)

$$
\xi_{\text {norm }}=\frac{\xi-\min \{z[n]\}}{\max \{z[n]\}-\min \{z[n]\}} .
$$

In [9], the kurtosis of received energy blocks $z[n]$ is employed to choose the appropriate normalized threshold. Kurtosis can be expressed as Eq. (11),

$$
k=\frac{1}{\left(N_{b}-1\right) \delta^{4}} \sum_{n=1}^{N_{\mathrm{b}}}(z[n]-\bar{z})^{4}-3,
$$

where $\bar{z}$ and $\delta$ denote the mean and standard deviation of $z[n]$ respectively. In this case, the ranging estimation can be expressed as Eq. (12)

$$
D=C \times \hat{t}_{T C}
$$

where $C$ is the speed of electromagnetic wave in the air and $t_{T C}$ denotes the TOA estimate of signal.

In a positioning system, the coordinates of the target nodes $(x, y, z)$ at the intersection of the circles of the reference nodes can be obtained by solving the following equations

$$
\left\{\begin{array}{c}
\sqrt{\left(X_{1}-x\right)^{2}+\left(Y_{1}-y\right)^{2}+\left(Z_{1}-z\right)^{2}} \\
\sqrt{\left(X_{2}-x\right)^{2}+\left(Y_{2}-y\right)^{2}+\left(Z_{2}-z\right)^{2}} \\
\cdots \cdots \\
\sqrt{\left(X_{k}-x\right)^{2}+\left(Y_{k}-y\right)^{2}+\left(Z_{k}-z\right)^{2}}
\end{array}\right\}=\left\{\begin{array}{c}
D_{1} \\
D_{2} \\
\cdots \\
D_{k}
\end{array}\right\},
$$

where $\left(X_{k}, Y_{k}, Z_{k}\right)$ is the coordinate of the $k$ th reference node, and $D_{k}$ is the range from the target node to the $k$ th reference node.

\section{The proposed threshold selection method}

\subsection{Low-frequency wavelet coefficient}

Wavelet transform has the characteristics of multiresolution analysis, and the received signal is divided into low-frequency part and high-frequency part using the wavelet decomposition. In the next layer process of wavelet decomposition, the low-frequency part is further divided into low-frequency part and high-frequency part, but the high-frequency part is no longer decomposed.

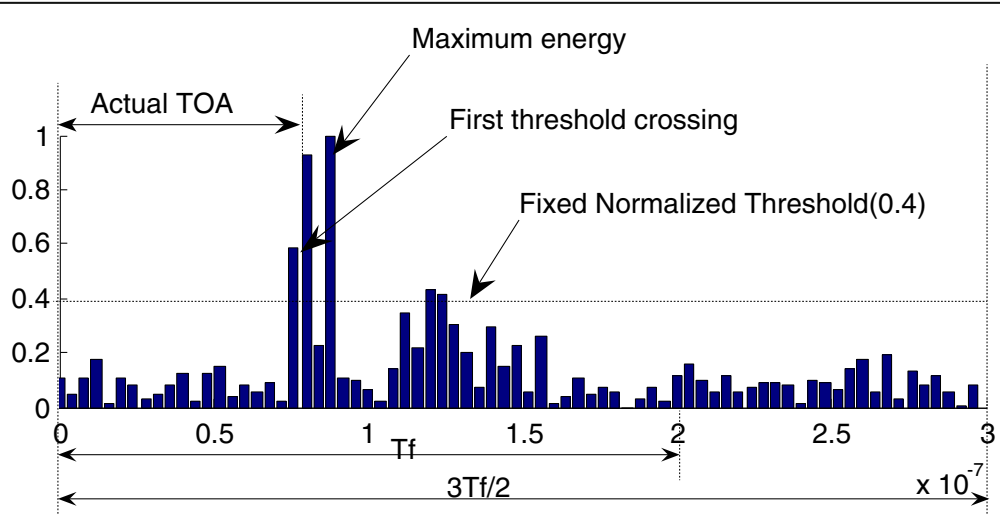

Fig. 2 TOA estimation techniques based on received energy 
Figure 3 shows a three-level wavelet decomposition tree. Multi-resolution analysis is used to decompose the low-frequency part of the signal to improve the resolution of the frequency. For the signal $S$, it is decomposed into high-frequency part $D 1$ and low-frequency part $A 1$, and then the low-frequency part of $A 1$ is further decomposed into high-frequency part $D 2$ and lowfrequency part $A 2$. And so on, the approximation part and detail part at any scales (resolutions) can be decomposed.

The above process of wavelet decomposition can be expressed as Eq. (14)

$$
\left\{\begin{array}{c}
c_{j+1}(k)=\sum_{n} h(n-2 k) c_{j}(n) \\
d_{j+1}(k)=\sum_{n} g(n-2 k) c_{j}(n),
\end{array}\right.
$$

where $c_{0}$ is the received UWB signal, $j \in\{0,1,2, \ldots\}$ denotes the levels of decomposition, $c_{j}$ is the lowfrequency coefficient using the $j$-level wavelet decomposition, and $d_{j}$ is the high-frequency coefficient using the $j$-level wavelet decomposition. $h(n)$ represents a lowpass filter, and $g(n)$ represents a high-pass filter, which are determined by the type of the selected wavelet.

In order to analyze the time domain waveform and energy spectrum of UWB, different values of $E_{b} /$ $N_{0}=\{40 \mathrm{~dB}, 35 \mathrm{~dB}, 30 \mathrm{~dB}\}$ are simulated, where $E_{b}$ is the energy in each bit and $N_{0}$ is one-sided power spectral density of additive white Gaussian noise. The parameters of the UWB transmission system are set as: $T_{f}=50 \mathrm{~ns}$, $T_{c}=1 \mathrm{~ns}, \varepsilon=0.5 \mathrm{~ns}$, sampling frequency $F_{c}=50 \mathrm{GHz}$, and transmission delay $=20$ ns. The simulation results are shown in Figs. 4, 5, and 6.

From the above simulations, the following conclusions can be drawn:

(1)Either in time domain or in frequency domain, when SNR decreases, the amplitude of the noise increases obviously comparing with the amplitude of the signal.

(2)The energy of signal is mainly distributed in the low frequency band, while the energy of white Gauss

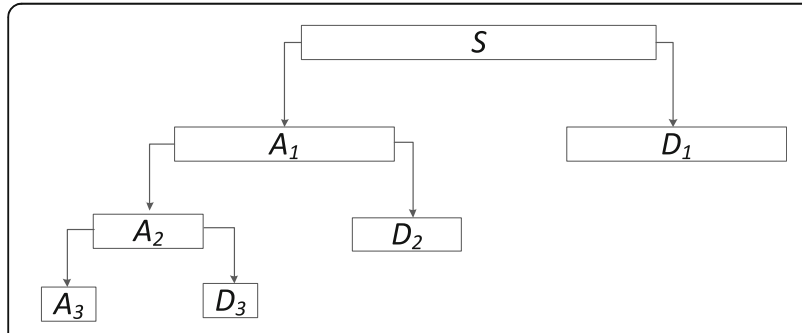

Fig. 3 Three-level wavelet decomposition

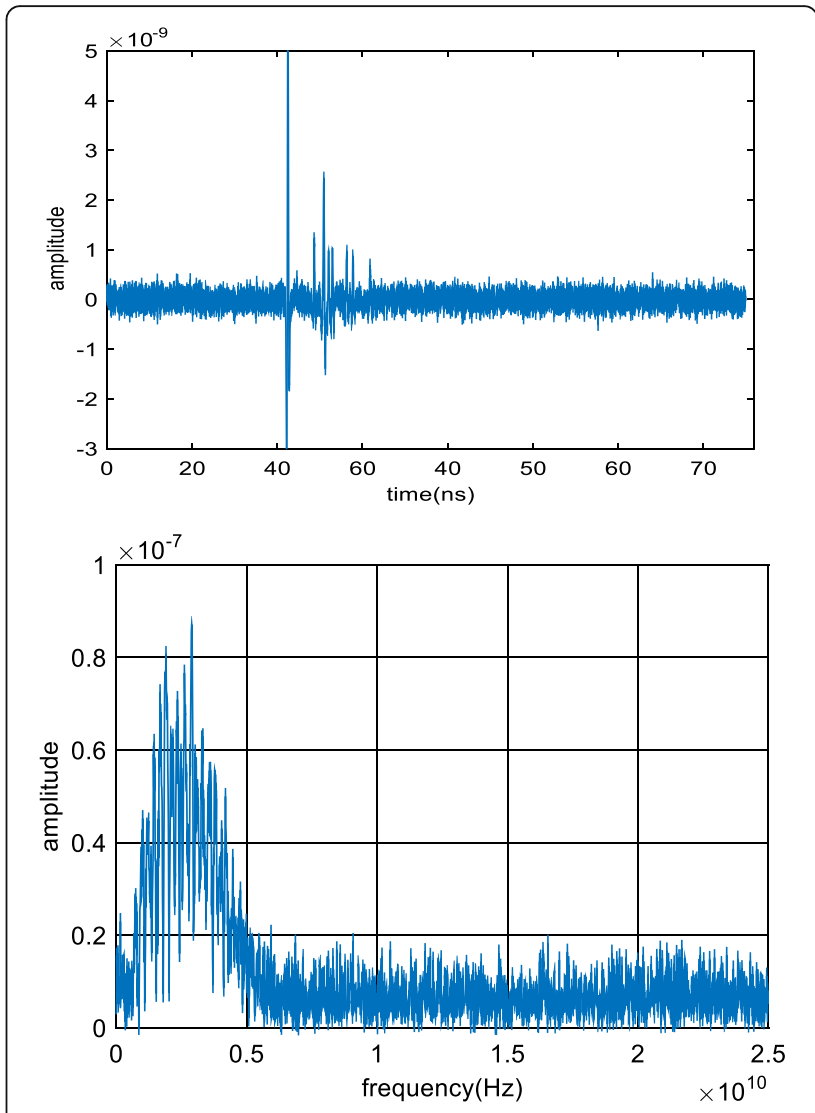

Fig. 4 Time domain waveform and energy spectrum with $E_{b} / N_{0}=40 \mathrm{~dB}$

noise is evenly distributed over the entire frequency band.

Because the wavelet decomposition is equivalent to two channel filter banks with low-pass and high-pass characteristics, after the wavelet decomposition, most energy of the received UWB signal concentrates in the coefficients of low-frequency bands, while the energy of the white Gauss noise distributes in the coefficients of different frequency bands. Therefore, in the energy detection receiver of this paper, after the wavelet transform, the high-frequency wavelet coefficients are discarded, and only the low-frequency wavelet coefficient is regarded as the received energy to improve the accuracy of ranging. In this way, the white Gauss noise interference in the signal can be reduced effectively.

\subsection{Kurtosis of the energy blocks of low-frequency coefficients}

In order to examine the characteristics of kurtosis of the energy blocks of the received signal $\left(K_{\mathrm{s}}\right)$ [9] and the kurtosis of the energy blocks of the decomposed lowfrequency wavelet coefficients $\left(K_{\mathrm{c}}\right)$, the CM1 (residential LOS) and CM2 (residential NLOS) channel models in 


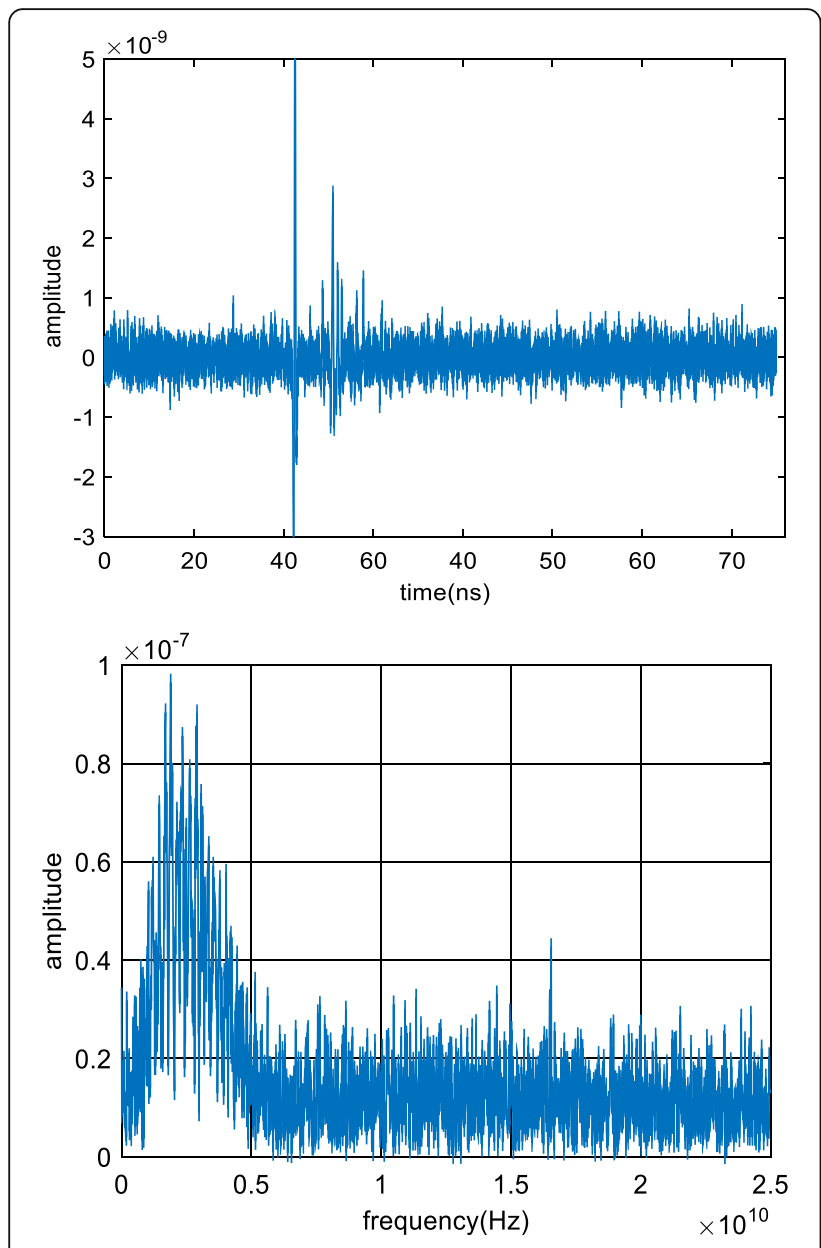

Fig. 5 Time domain waveform and energy spectrum with $E_{b} / N_{0}=35 \mathrm{~dB}$

the IEEE802.15.4a standard are employed. $K_{\mathrm{s}}$ can be expressed as Eq. (15),

$$
K_{s}=\frac{1}{\left(N_{s}-1\right) \delta^{4}} \sum_{n=1}^{N_{s}}\left(Z_{s}[n]-\overline{Z_{s}}\right)^{4}-3
$$

where $Z_{s}[n]$ is the received signal energy blocks, $\overline{Z_{s}}$ and $\delta$ denote the mean and standard deviation of $Z_{s}[n]$ respectively. The number of samples in each energy integration interval is represented as $N_{\mathrm{i}}=T_{\mathrm{b}} \times F_{\mathrm{c}}$, where $T_{\mathrm{b}}$ is the integration period, and $F_{\mathrm{c}}$ is the sampling frequency. The number of signal samples in each frame is represented as $N_{\mathrm{f}}=T_{\mathrm{f}} \times F_{\mathrm{c}}$, where $T_{\mathrm{f}}$ is the frame period. The number of energy blocks within a frame is $N_{s}=\left\lfloor\frac{N_{f}}{N_{i}}\right\rfloor=N_{b}$, where \lfloor\rfloor denotes the integer part.

$K_{\mathrm{c}}$ can be expressed as Eq. (16),

$$
K_{c}=\frac{1}{\left(N_{c}-1\right) \delta^{4}} \sum_{n=1}^{N_{c}}\left(Z_{c}[n] \overline{Z_{c}}\right)^{4}-3,
$$

where $Z_{c}[n]$ is the energy blocks of the decomposed low-frequency coefficients, $\bar{Z}$ and $\delta$ denote the mean

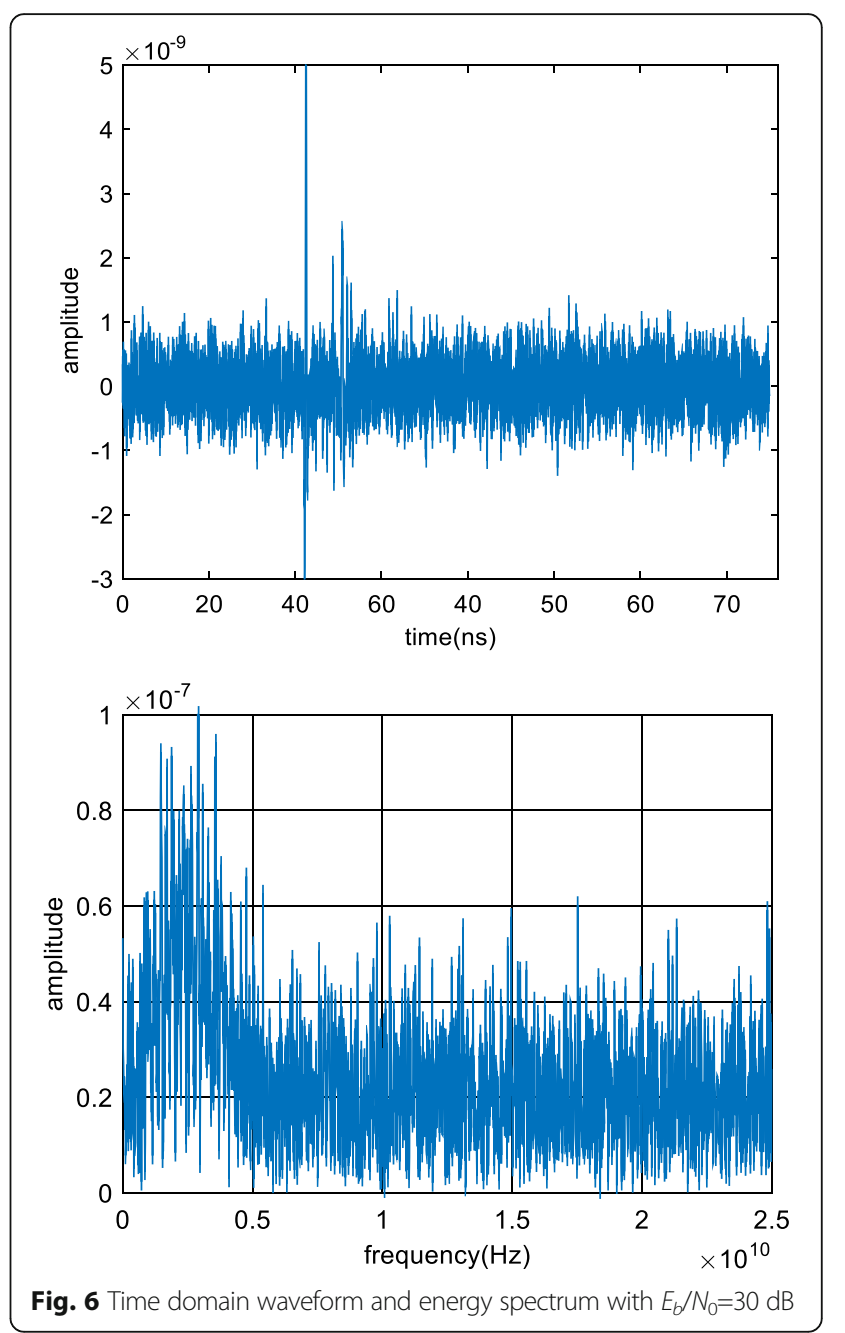

and standard deviation of $Z_{c}[n]$ respectively. The number of the low-frequency coefficients in each integration interval $N_{i c}$ is represented as Eq. (17)

$$
N_{i c}=\left\lfloor\mathrm{T}_{b} \times F_{c} \times \frac{N_{f c}}{N_{f}}\right\rfloor,
$$

where $N_{f c}$ is the number of the low-frequency coefficients in each frame. And then the number of the energy blocks of low-frequency coefficients is Eq. (18)

$$
N_{c}=\left\lfloor\frac{N_{f c}}{N_{i c}}\right\rfloor \text {. }
$$

For each $E_{b} / N_{0}$ value of $\{5 \mathrm{~dB}, 6 \mathrm{~dB}, \ldots, 25 \mathrm{~dB}\}, 1000$ channel realizations were generated with $F_{\mathrm{c}}=50 \mathrm{GHz}$. The second derivative Gaussian pulse was employed with $T_{\mathrm{b}}=0.2 \mathrm{~ns}, T_{f}=50 \mathrm{~ns}$, and $N_{s}=1$. The "db6" wavelet with two-layer decomposition was used. The simulation of average $K_{\mathrm{s}}$ and $K_{\mathrm{c}}$ are as shown in Figs. 7 and 8 .

Figures 7 and 8 illustrate that the characteristics of $K_{\mathrm{s}}$ and $K_{\mathrm{c}}$ with respect to different values of $E_{b} / N_{0}$ is almost 


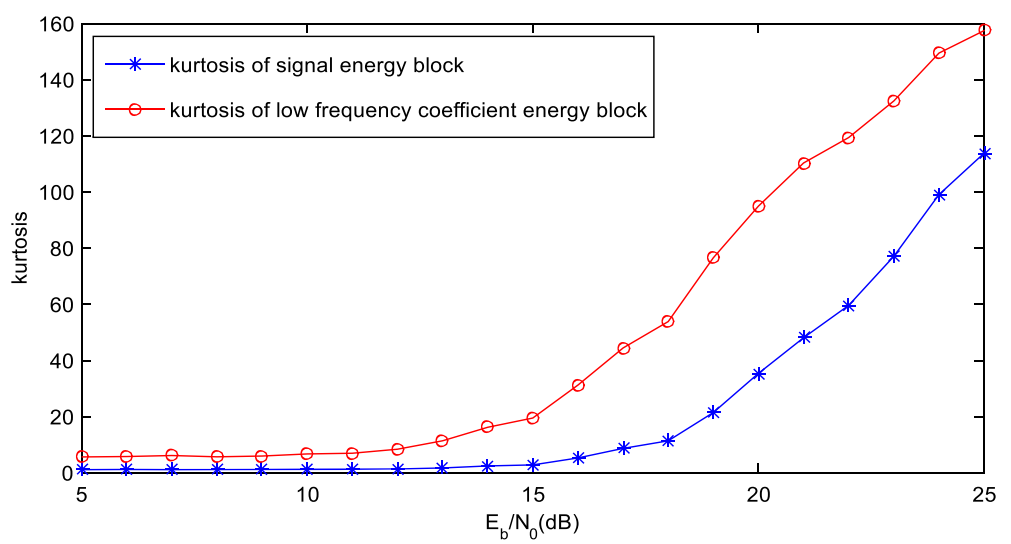

Fig. $7 K_{5}$ and $K_{c}$ in $\mathrm{CM} 1$

the same for the two different channels. Furthermore, Figs. 7 and 8 show that $K_{\mathrm{s}}$ and $K_{\mathrm{c}}$ increase as $E_{b} / N_{0}$ increases, but $K_{\mathrm{c}}$ changes more rapidly. Therefore, $K_{\mathrm{c}}$ can better reflect the changes with different values of $E_{b} / N_{0}$ and it is more suitable for threshold selection.

\subsection{Relationship between $\mathrm{K}_{\mathrm{c}}$ and threshold}

To determine the optimal threshold $\xi_{\text {best }}$ based on $K_{\mathrm{c}}$, the relationship between the MAE, $K_{\mathrm{c}}$, and the normalized threshold $\xi_{\text {norm }}$ for different $K_{\mathrm{c}}$ was determined. One thousand channel realizations with different values of $E_{\mathrm{b}} / N_{0}$ were simulated. There are four steps to establish the relationship:

(1) Generate a large amount of receive signals for each channel model under different $E_{\mathrm{b}} / N_{0}$ values of $\{5 \mathrm{~dB}$, $6 \mathrm{~dB}, \ldots, 25 \mathrm{~dB}\}$.

(2) Calculate the average MAE values with respect to different normalized thresholds $\xi_{\text {norm }}$ of $\{0.1,0.2, \ldots$,
$1.0\}$ for each $K_{\mathrm{c}}$ value. With each channel realization, the thresholds are compared with $Z_{c}[n]$ to find the first sample index crossing the normalized threshold, as shown in Eq. (10). In the simulation, because of the random noise signal, there are different MAE values with respect to one specific normalized threshold and one specific $E_{\mathrm{b}} / N_{0}$ value, so the average MAE is calculated with respect to one specific normalized threshold. Moreover, because $K_{\mathrm{c}}$ is a real value, $K_{\mathrm{c}}$ is rounded to the nearest integer value.

(3) Select the normalized threshold with the lowest MAE as the best threshold $\xi_{\text {best }}$ with respect to specific $K_{\mathrm{c}}$ for each channel model.

(4)A polynomial with degree 3 is fitted to the best threshold $\xi_{\text {best }}$ for each value of $K_{\mathrm{c}}$ by using the method of least-squares where $K_{\mathrm{c}}$ is the $x$-coordinate and $\xi_{\text {best }}$ is the $y$-coordinate. To obtain the coefficient estimates, the least-squares method minimizes the summed square of residuals. The

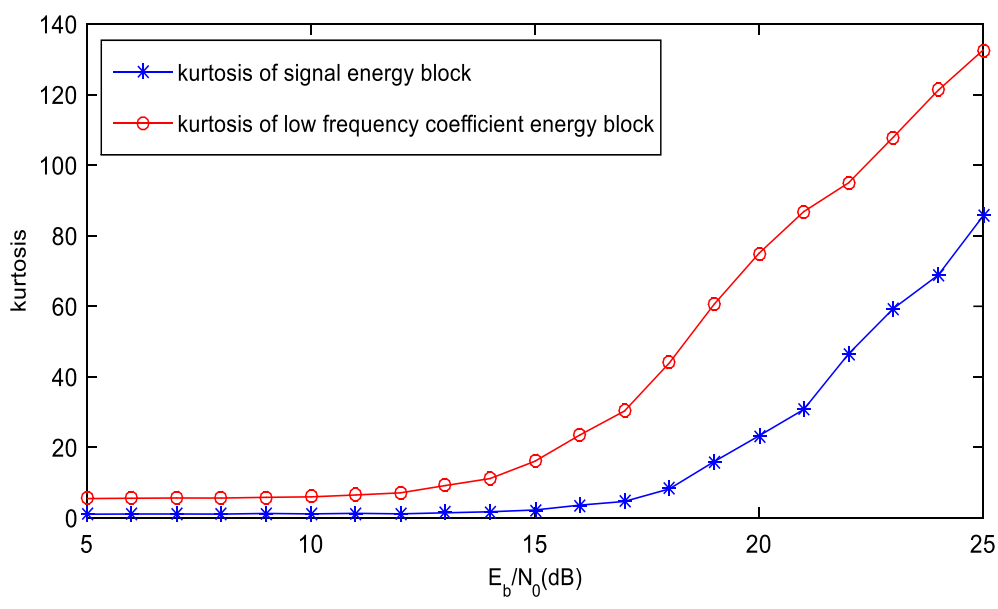

Fig. $8 K_{s}$ and $K_{c}$ in $\mathrm{CM} 2$ 
$i$ th residual $r_{i}$ for the $i$ th pair of $\left(K_{\mathrm{c}}, \xi_{\text {best }}\right)$ is defined as

$$
r_{i}=y_{i}-\hat{y}_{i}
$$

where $y_{i}$ is the best threshold and $\hat{y}_{i}$ is the fitted threshold value for the $i$ th $K_{\mathrm{c}}$, so the summed square of residuals $S_{\mathrm{K}}$ is given by

$$
S_{K}=\sum_{i=1}^{n} r_{i}^{2}=\sum_{i=1}^{n}\left(y_{i}-\hat{y}_{i}\right)^{2}
$$

where $n$ is the number of $\left(K_{\mathrm{c}}, \xi_{\text {best }}\right)$. The fitting result based on $K_{\mathrm{c}}$ are shown in Eq. (21)

$\mathrm{CM} 1: \xi_{c 1}=-3.1079 \times 10^{-8} K_{c}^{3}+2.5191 \times 10^{-5} K_{c}{ }^{2}-7.0462 \times 10^{-3}$

$$
\times K_{c}+0.82585
$$

$\mathrm{CM} 2: \xi_{c 1}=-2.6988 \times 10^{-8} K_{c}{ }^{3}+2.2681 \times 10^{-5} K_{c}{ }^{2}-6.5214 \times 10^{-3}$

$$
\times K_{c}+0.7816 .
$$

where $\xi_{\mathrm{c} 1}$ and $\xi_{\mathrm{c} 1}$ are the optimal thresholds for CM1 and $\mathrm{CM} 2$.

\section{Performance results and discussion}

\subsection{TOA estimation error}

In order to compare with the method without wavelet decomposition [9], the optimal thresholds $\xi_{\mathrm{s} 1}$ and $\xi_{\mathrm{s} 1}$ for CM1 channel and CM2 channel based on $K_{\mathrm{s}}$ are generated as Eq. (22) using the similar method as shown in [9].

$$
\begin{aligned}
& \mathrm{CM} 1: \xi_{s 1}=-8.0514 \times 10^{-8} K_{s}{ }^{3}+4.5232 \times 10^{-5} K_{s}{ }^{2}-8.7546 \times 10^{-3} \\
& \times K_{s}+0.78051 \\
& \mathrm{CM} 2: \xi_{s 1}=-7.7291 \times 10^{-8} K_{s}^{3}+4.4374 \times 10^{-5} K_{s}^{2}-8.7246 \times 10^{-3}
\end{aligned}
$$$$
\times K_{s}+0.76794
$$

In this section, the MAE is examined for $K_{\mathrm{c}}$-based and $K_{\mathrm{s}}$-based threshold selection algorithms in the CM1 and CM2 channel models of IEEE 802.15.4a standards. As before, 1000 channel realizations were simulated for each case. The performance results are shown in Figs. 9 and 10.

Figures 9 and 10 present the following:

(1)In CM1 and CM2, the MAE of the $K_{\mathrm{c}^{-}}$-based and $K_{\mathrm{s}}$ based threshold selection methods decrease as the $E_{b} / N_{0}$ increase. But when $E_{\mathrm{b}} / N_{0}$ is between 9 and $20 \mathrm{~dB}$, the proposed method is better than that of the $K_{\mathrm{s}}$-based method proposed in [9]. It can be found that in CM1 channel, when $E_{\mathrm{b}} / N_{0}$ is $14 \mathrm{~dB}$, the MAE of $K_{\mathrm{c}}$-based method is about 5 ns better than that of the $K_{\mathrm{s}}$-based method. In CM2 channel, when $E_{\mathrm{b}} / N_{0}$ is $15 \mathrm{~dB}$, MAE of $K_{\mathrm{c}}$-based method is

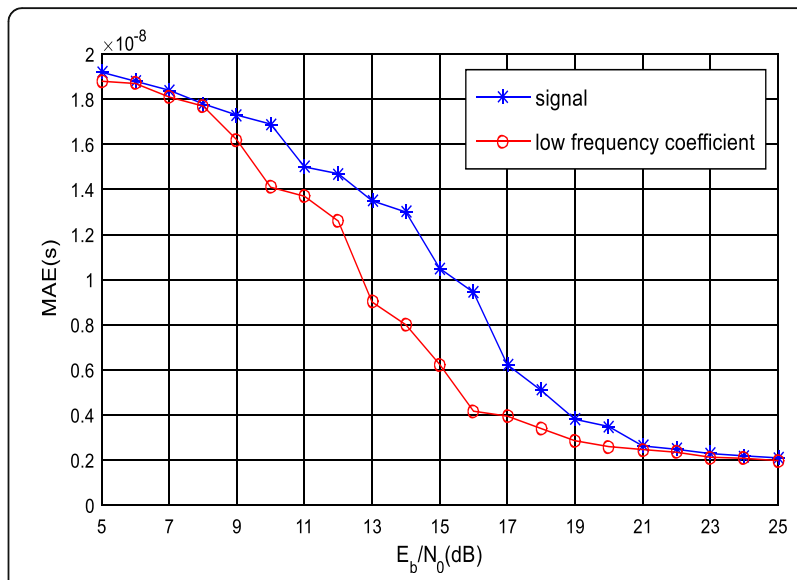

Fig. $9 M A E$ in $C M 1$

about 5.87 ns better than that of the $K_{\mathrm{s}}$-based method.

(2) When $E_{\mathrm{b}} / N_{0}$ is less than $8 \mathrm{~dB}$, the MAE of the two algorithms are very near. This is because now the energy of the noise is very high, which will affect the decomposed low-frequency coefficients seriously, so the advantage of the proposed method is little compared with the $K_{\mathrm{s}}$-based method.

(3) When $E_{\mathrm{b}} / N_{0}$ is higher than $21 \mathrm{~dB}$, the MAE of the two methods is almost the same. This is because now the energy of the noise is very low compared with the signal energy, which will not affect the two methods.

\subsection{Computational complexity}

In order to compare the computational complexity of different methods, 100 iterations were performed for each $E_{b} / N_{0}$ value of $\{5 \mathrm{~dB}, 6 \mathrm{~dB}, \ldots, 25 \mathrm{~dB}\}$, and the total running time of $100 \times 25=2100$ iterations is given in Table 3. In the simulation, the amount of energy blocks of $K_{\mathrm{c}}$ is 472 and for $K_{\mathrm{s}}$ is 375 . At the same time, the

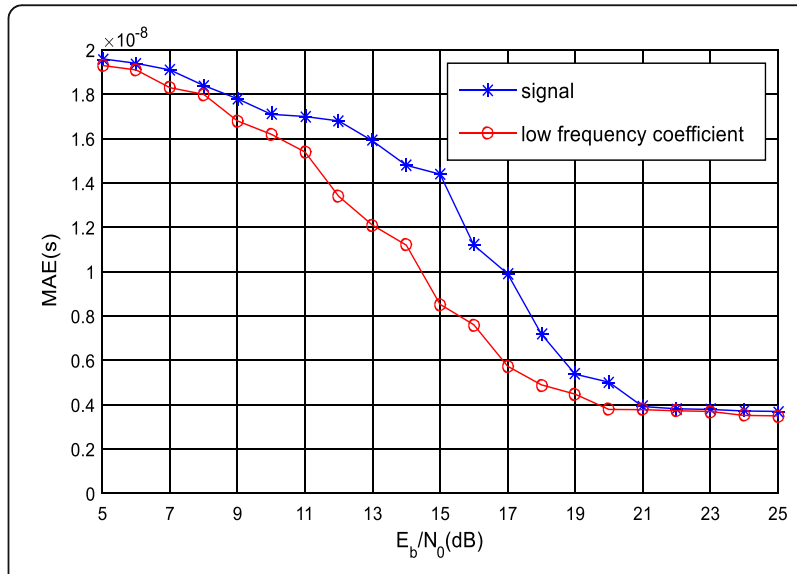

Fig. 10 MAE in CM2 
Table 3 Running time of different methods

\begin{tabular}{llllllllll}
\hline $\begin{array}{l}\text { Operation } \\
(2100 \\
\text { iterations) }\end{array}$ & Energy block & $\begin{array}{l}\text { Kurtosis } \\
\text { analysis }\end{array}$ & $\begin{array}{l}\text { TOA } \\
\text { estimation }\end{array}$ & Total & \\
\hline Methods & $K_{c}$ & $K_{s}$ & $K_{c}$ & $K_{s}$ & $K_{c}$ & $K_{s}$ & $K_{c}$ & $K_{s}$ \\
Time (s) & 22.581 & 18.219 & 0.451 & 0.390 & 0.241 & 0.248 & 23.582 & 19.188 \\
\hline
\end{tabular}

signal decomposed using wavelet transform needs extra computation, so the running time of the $K_{\mathrm{c}}$ method is a little higher than that of the $K_{\mathrm{s}}$ method. For Each TOA estimation process, the difference of running time is just $2 \mathrm{~ms}$, so this is acceptable.

\section{Conclusions}

In the UWB ranging system, the energy detection method based on non-coherent receiver is widely used. However, because of the interference, such as multi-path fading, thermal noise, inter-symbol interference, and reflection interference, the precision of ranging is not very high. The simulation results show that in the UWB signal decomposed by wavelet transform, most of the signal energy is concentrated in the low-frequency band, while the energy of noise is evenly distributed over the entire frequency band. Therefore, this paper proposes a new threshold selection method based on wavelet decomposition and kurtosis analysis, that is, the UWB signal is decomposed by wavelet transform, the threshold is obtained based on the kurtosis of energy blocks of low-frequency wavelet coefficient, and then the first energy block exceeding the threshold is treated as the TOA of signal. The simulation results show that the new method can obviously improve the precision of TOA estimation.

\section{Acknowledgements}

This work was supported by the National Natural Science Foundation of China under Grant No. 61671482, the Nature Science Foundation of Shandong Province No. ZR2014FL014, and the Fundamental Research Funds for the Central Universities Nos. 16CX02046A, 17CX02042A, and 14CX02212A.

\section{Authors' contributions}

The authors have contributed jointly to all parts on the preparation of this manuscript, and all authors read and approved the final manuscript.

\section{Competing interests}

The authors declare that they have no competing interests.

\section{Publisher's Note}

Springer Nature remains neutral with regard to jurisdictional claims in published maps and institutional affiliations.

\section{Author details}

'Department of Computer and Communication Engineering, China University of Petroleum (East China), Qingdao 266580, China. ${ }^{2}$ Department of Electrical, Computer, Software, and Systems Engineering, Embry-Riddle Aeronautical University, Daytona Beach, FL 32114, USA.
Received: 27 August 2017 Accepted: 15 November 2017

Published online: 28 November 2017

\section{References}

1. X Zheng, Z Cai, J Li, H Gao, A study on application-aware scheduling in wireless networks. IEEE Trans. Mob. Comput. 16(7), 1787-1801 (2017). https://doi.org/10.1109/TMC.2016.2613529

2. S Cheng, Z Cai, J Li, Curve query processing in wireless sensor networks. IEEE Trans. Veh. Technol. 64(11), 5198-5209 (2015). https://doi.org/10.1109/ TVT.2014.2375330

3. V Savic, EG Larsson, J Ferrer-Coll, P Stenumgaard, Kernel methods for accurate UWB-based ranging with reduced complexity. IEEE Trans. Wirel. Commun. 15(3), 1783-1793 (2016). https://doi.org/10.1109/TWC.2015. 2496584

4. F Simone, R Francescantonio Della, in mobile positioning and tracking: from conventional to cooperative techniques. Fundamentals of positioning (Wiley-IEEE Press, New Jersey, United States, 2017), p. 416

5. PK Yoon, S Zihajehzadeh, BS Kang, EJ Park, Robust biomechanical modelbased 3-D indoor localization and tracking method using UWB and IMU. IEEE Sensors J. 17(4), 1084-1096 (2017). https://doi.org/10.1109/JSEN.2016. 2639530

6. Z He, Z Cai, S Cheng, X Wang, Approximate aggregation for tracking quantiles and range countings in wireless sensor networks. Theor. Comput. Sci. 607, 381-390 (2015). https://doi.org/10.1016/j.tcs.2015.07.056

7. MZ Win, RA Scholtz, Ultra-wide bandwidth time-hopping spread-spectrum impulse radio for wireless multiple-access communications. IEEE Trans. Commun. 48(4), 679-689 (2000). https://doi.org/10.1109/26.843135

8. I Guvenc, Z Sahinoglu, in ICU 2005: 2005 IEEE International Conference on Ultra-Wideband. Threshold-based TOA estimation for impulse radio UWB systems (Switzerland, Zurich, 2005), pp. 420-425. https://doi.org/10.1109/ ICU.2005.1570024

9. I Guvenc, Z Sahinoglu, Threshold selection for UWB TOA estimation based on kurtosis analysis. IEEE Commun. Lett. 9(12), 1025-1027 (2005). https://doi. org/10.1109/LCOMM.2005.1576576

10. S Cheng, Z Cai, J Li, X Fang, in 2015 IEEE Conference on Computer Communications (INFOCOM). Drawing dominant dataset from big sensory data in wireless sensor networks (2015), pp. 531-539. https://doi.org/10. 1109/INFOCOM.2015.7218420

11. J Li, S Cheng, Y Li, Z Cai, in 2015 IEEE 35th International Conference on Distributed Computing Systems. Approximate holistic aggregation in wireless sensor networks (2015), pp. 740-741. https://doi.org/10.1109/ICDCS.2015.86

12. S Cheng, Z Cai, J Li, H Gao, Extracting kernel dataset from big sensory data in wireless sensor networks. IEEE Trans. Knowl. Data Eng. 29(4), 813-827 (2017). https://doi.org/10.1109/TKDE.2016.2645212

13. Z Xiao, H Wen, A Markham, N Trigoni, P Blunsom, J Frolik, Non-line-of-sight identification and mitigation using received signal strength. IEEE Trans. Wirel. Commun. 14(3), 1689-1702 (2015). https://doi.org/10.1109/TWC.2014.2372341

14. E Arias-de-Reyna, JJ Murillo-Fuentes, R Boloix-Tortosa, Blind low complexity time-of-arrival estimation algorithm for UWB signals. IEEE Signal Processing Letters 22(11), 2019-2023 (2015). https://doi.org/10.1109/LSP.2015.2450999

15. V Savic, J Ferrer-Coll, P Ängskog, J Chilo, P Stenumgaard, EG Larsson, Measurement analysis and channel modeling for TOA-based ranging in tunnels. IEEE Trans. Wirel. Commun. 14(1), 456-467 (2015). https://doi.org/ 10.1109/TWC.2014.2350493

16. A Jafari, T Mavridis, L Petrillo, J Sarrazin, M Peter, W Keusgen, PD Doncker, A Benlarbi-Delai, UWB Interferometry TDOA estimation for 60-GHz OFDM communication systems. IEEE Antennas and Wireless Propagation Letters 15, 1438-1441 (2016). https://doi.org/10.1109/LAWP.2015.2512327

17. BG Yu, G Lee, HG Han, WS Ra, TW Kim, A time-based angle-of-arrival sensor using CMOS IR-UWB transceivers. IEEE Sensors J. 16(14), 5563-5571 (2016). https://doi.org/10.1109/JSEN.2016.2567441

18. S Büyükçorak, T Erbaş, GK Kurt, A Yongaçoğlu, in 2014 22nd Signal Processing and Communications Applications Conference (SIU). Indoor localization applications (2014), pp. 1239-1242. https://doi.org/10.1109/SIU.2014.6830460

19. GD Angelis, A Moschitta, P Carbone, Positioning techniques in indoor environments based on stochastic modeling of UWB round-trip-time measurements. IEEE Trans. Intell. Transp. Syst. 17(8), 2272-2281 (2016). https://doi.org/10.1109/TITS.2016.2516822

20. AYZ Xu, A E K S, W A K S, W Qin, A novel threshold-based coherent TOA estimation for IR-UWB systems. IEEE Trans. Veh. Technol. 58(8), 4675-4681 (2009). https://doi.org/10.1109/TVT.2009.2020990 
21. X Cui, H Zhang, TA Gulliver, Threshold selection for ultra-wideband TOA estimation based on neural networks. Journal of Networks 7(9), 1311-1318 (2012). https://doi.org/10.4304/jnw.7.9.1311-1318

22. A F Molisch, Balakrishnan K, Cassioli D, Chong C-C, Emami S, Fort A, Karedal J, Kunisch J, Schantz H, Schuster U, Siwiak K. IEEE 802.15.4a channel model-final report, http://www.ieee802.org/15/pub/04/15-04-0662-02004a-channel-model-final-report-r1.pdf. Accessed July 302017

\section{Submit your manuscript to a SpringerOpen ${ }^{\bullet}$ journal and benefit from:}

- Convenient online submission

- Rigorous peer review

- Open access: articles freely available online

- High visibility within the field

- Retaining the copyright to your article

Submit your next manuscript at $\gg$ springeropen.com 